\title{
LA EVALUACIÓN DEL RENDIMIENTO ACADÉMICO $Y$ SU RELACIÓN CON EL MODELO DE FORMACIÓN POR COMPETENCIAS
}

KAROL. STEFANÍA AMAYA GARCÍA Magister

Maestría en Educación

Universidad Surcolombiana Septiembre de 2013

La iniciativa investigativa parte desde lo regulado por el Decreto 1295 de 2010 Capitulo lif numeral 5.3-5.3.2, por medio del cual se exige a las Instituciones de Educación Superior en Colombia formar desde el enfoque por competencias. A partir de éste discurso pedagógico oficial las instituciones iniciaron una re-estructuración de los diseños curriculares, procesos, prácticas y agentes involucrados en el proceso formativo con el propósito de responder a| logro de esta nueva formación. En consecuencia, se supone que la evaluación, como elemento inherente en el proceso de formación del estudiante, hizo parte de dicha re-estructuración.

Pero es evidente la situación actual de la evaluación en las aulas cle clases, pues se observa que ésta no tiene relación con el nuevo modelo formativo, dado que nose da una coherencia entre el discurso y la acción. La comple jidad del proceso evaluativo radica en medir la transmisión de conocimiento deł docente hacia el estudiante, a través del parcial como única estrategia para hacer seguimiento a los aprendizajes. Es decir, en la mayoría de los casos, las evaluaciones están diseñadas para męir sólo los conocimientos teóricos, situación que se presenta en la medida en que los docentes perciben la evaluación como un proceso de medición de aprendizaje que se debe realizar periódicamente para llevar un registro de los estudiantes que aprueban un curso.

La aplicación de este tipo de evaluación da lugar a que el estudiante se acostumbre a concentrar sus esfuerzos para pasar el curso, generando así un aprendizaje memorístico y sin verdadero significado. A lo anterior se suma el uso de la evaluación como instrumento de poder y control en la medida en que su resultado genera una segregación y una división fuertemente marcada entre los éxitos y los fracasos, lo cual advierte gue no existe ninguna neutralidad valorativa en el proceso de evaluación sino, por el contrario, hay una intencionalidad declarada tàcita en dichos procesos.

Además, en la mayoría de los casos, las evaluaciones están diseñadas para medir sólo los conocimientos teóricos con un énfasis marcado en una percepción instrumental y algoritmica de la evaluación, situación que se presenta en la medida en que los docentes perciben la evaluación como un proceso de medición de aprendizajes gue se debe realizar periódicamente para llevar un registro de los estudiantes que aprueban un curso. Desde esta perspectiva el docente se concibe como protagonista del proceso formativo y el estudiante como receptor de conoc imientos. 
La anterior problemática parte de la falta de formación y preparación de los docentes sobre la formación por competencias; quiere esto decir que los docentes presentan vacíos conceptuales y metodológicos que han permitido que continúen con su modelo implícito (modelo tradicional), el cual de termina sus prácticas evaluativas. Es evidente que al darse esta situación nose desarrollan prácticas pedagógicas y evaluativas coherentes con el tipo de profesional que la Universidad pretende formar.

Ante esta postura se puede deducir que la evaluación está lejos de ser percibida como una oportunidad para promover y mejorar los aprendizajes de los estudiantes. La formación por competencias exige una re-estructuración del proceso de evaluación, y éste va más allá de su enunciación en el Proyecto Educativo Universitario. La anterior situación es evidenciada en la universidad Surcolombiana y en especial en la Facultad de Educación, en donde las evaluaciones escritas de memorización continúan dominando en muchos programas académicos.

Cómo la formación profesional vincula tres dispositivos que se encuentran unidos de forma inseparable (las estructuras curriculares, las prácticas pedagógicas y los procesos evaluativos) al presentarse una incoherencia entre los mismos se genera una ruptura en el proceso formativo del estudiante generando unos bajos niveles de calidad, además de presentarse ausencia de pertenencia social y pertinencia académica del proceso, dado que los diseños curriculares se elaboran a partir de un perfil de formación requerido y las prácticas evaluativas se realizan desde otra perspectiva diferente.

Cuando se forma por competencias y se evalúa a partir de otro modelo formativo, se presenta un problema en cuanto al aprendizaje de los estudiantes, toda vez que los resultados de una evaluación deben permitir evidenciar los aprendizajes obtenidos por los estudiantes y las falencias que se presentan para el cumplimiento de los mismos; por esta razón, debe haber una coherencia entre los dos procesos que permita conocer si se alcanzaron los logros establecidos en la formación del profesional. Frente a esta situación no es claro cómo se relaciona el proceso evaluativo que se lleva a cabo en el aula de clases de la Facultad de Educación con el proceso de formación planteado en el Proyecto Educativo Universitario y con los propósitos de formación de la Facultad.

Desde esta perspectiva se formuló la siguiente pregunta que dio lugar al desarrollo del proyecto de investigación: ¿Qué tipo de relación existe entre el proceso evaluativo que realizan los docentes para la formación del profesional de la Facultad de Educación y el modelo de formación por competencias de la Universidad Surcolombiana?

Partiendo del anterior contexto, se hizo necesario fijar los objetivos del trabajo investigativo, dado a que serian éstos los orientadores del desarrollo del mismo. Por tanto se definió como objetivo general caracterizar la relación existente entre el proceso evaluativo que se implementa actualmente en la formación del profesional de la Facultad de Educación de $\mathbf{a}$ Univer sidad Surcolombiana con el modelo de formación por competencias; y como ob jetivos específicos identificar las estrategias de evaluación que actualmente se desarrollan en el aula de clases de la Facultad de Educación de la Universidad Surcolombiana; determinar la postura quetienen los directivos, los docentes y los estudiantes de la Facult ad de Educación frente a los conceptos de evaluación y evaluación por competencias; Establecer la relación 
entre la evaluación y la formación por competencias que permita idẹtịificar su grado de coherencia e incoherencia; y plantear una alternativa de intervención o cambio de la realidad encontrada que permita a la Universidad Surcolombiana contar con herramientas fundamentales para intervenir el proceso formativo de los estudiantes de Facultad de Educación.

Como era necesario fundamentar el trabajo con referentes teóricos e investigativos, se elaboró un marco de referencia que recogió elementos de la educación superior en el siglo XXI, acerca de la educación superior en Colombia, información sobre la Universidad Surcolombiana y especificamente de a Facultad de Educación; el concepto de competencia; la formación desde el enfoque por competencias; la evaluación del rendimiento académico; la evaluación por competencias; los tipos de evaluación; las pruebas saber pro; y finalmente sobre la evaluación del rendimiento académico en la Universidad Surcolombiana.

Para el diseño metodológico que orientaría el trabajo de campo de la investigación se llevó a cabo una investigación etnográfica mediante un enfoque cualitativo, entendiendo la etnografía como "un método de investigación que permite describir, interpretar y analizar un ámbito sociocultural especifico con el fin de conocer sus vivencias, percepciones, opiniones y experiencias" (Ana Lía Kornblitt : 2004).

A partir de lo anterior, el estudio de la problemática implicó un proceso de descripción en donde se intentó presentar la realidad de la misma desde las propias acciones de los actores (experiencias, creencias, actitudes, pensamientos, reflexiones), ya que se interactuó con cada uno de ellos (docentes, estudiantes y directivos de la Universidad Surcolombiana) mediante un acercamiento y comunicación permanentes, de tal forma que se pudiera conocer la realidad de sus contextos. La información obtenida se convirtió en elementos fundamentales para la construcción de los análisis.

Desde esta perspectiva se llevó a cabo un análisis de la relación del proceso evaluativo que se implementa en la formación del profesional de la Facultad de Educación de la Universidad Surcolombiana con el modelo de formación por competencias, que permitió construir una base argumentativa para la caracterización de la realidad actual sustentada y fundamentada en acciones de cambio o transformación que se considera conveniente realizar en los procesos de evaluación de la Facultad de Educación.

La investigación desarrollada permitió realizar una indagación sistematica de los planteamientos y puntos de vista de directivos, docentes y estudiantes; un procesamiento de la información obtenida a partir de los objetivos propuestos y el análisis de los actuales procesos de evaluación en la Facultad de Educación.

Es necesario destacar que durante el análisis y procesamiento de la información se recurrió al uso de algunas cifras o datos numéricos que permitieron la comprensión de la problemática investigada, sin que esto se convir tiera en el elemento central del análisis y la comprensión de los sujetos y la realidad de la que hacen parte. Es decir, la cantidad o cifra asume un papel secundario en esta investigación lo que orienta el análisis es determinar las concepciones, la cosmovisión, las razones de la relación entre la formación por competencias y la evaluación por competencias. 
En lo concerniente a la población y muestra, la investigación se desarrolló en la Facultad de Educación de la Universidad Surcolombiana, con la comunidad académica que la integra, la cual fue objeto de estudio y análisis. Se aplicó cuestionarios a ciento cincuenta (150) de ellos, pertenecientes a los siete programas académicos, los cuales representan una muestra significativa del total de los estudiantes de pregrado de la Facultad de Educación equivalente al $10 \%$, porque se considera importante rescatar la voz de los actores. De igual forma hicieron parte de la muestra veinte (20) docentes pertenecientes a la Facultad de Educación, con modalidad de vinculación catedráticos, docentes de planta, tiempo completo y medio tiempo. El criterio que se utilizó para seleccionar los docentes fue ser docentes con más de tres (3) años de trabajo académico en la Universidad.

Para conocer las posturas de los diferentes entes reguladores de los asuntos académicos de la Facultad de Educación y de la Universidad se entrevistó a la Decana de la Facultad, e Director General de Currículo, a Vicerrector Académico y al Rector.

Para la obtención de información se utilizó una batería de instrumentos compuesta por entrevistas estructuradas, cuestionarios con preguntas abiertas, y el análisis de documentos; lo que garantizó la recolección de información relacionada con aspectos sustantivos de la evaluación del rendimiento académico y del proceso de formación por competencias.

Una vez realizada la aplicación de los instrumentos de investigación, se organizó y analizó la información utilizando tres categorías que permitieron abordar con claridad y validez la problemática de estudio y alcanzar los objetivos propuestos de la investigación. Las categorías fueron denominadas de la siguiente manera: Evaluación del rendimiento académico; Competencias y Formación del profesional de la Facultad de Educación.

La organización y análisis de la información permitió encontrar que los programas de pregrado de la Facultad de Educación mencionan que están formando por competencias tal como b exige el Decreto 1295 de 2010.

Así mismo, la Univer sidad Surcolombiana actualmente forma a los profesionales de la educación bajo un modelo pedagógico tradicional, muy ligado al aprendizaje memorístico, la transmisión de conocimientos, con los roles previamente establecidos, en donde el modelo curricular está organizado en áreas açadémicas y asignaturas. Dicho modelo concibe al docente como protagonista del proceso y al estudiante como receptor de conocimientos; además de manejar un concepto genérico del mismo, es decir, todos los estudiantes son iguales. Esta práctica pedagógica previene de unas representaciones que el docente ha construido desde la perspectiva de pensar queen la universidad se trata de poner en circulación los saberes propios de cada área o disciplina.

Los programas académicos de la Facultad de Educación en su Proyecto Educativo de Programa, señalan estar ofreciendo una formación a través del enfoque de competencias, queen la realidad se queda sólo en la enunciación del mismo. Este rediseño de los modelos curriculares de la Facultad de Educación ha obedecido a exigencias legales emanadas del Ministerio de Educación Nacional y del ICFES, para el funcionamiento y acreditación de los mismos, los cuales no tienen ninguna relación con las prácticas pedagógicas de los docentes. 
Mientras subsista la transmisión como paradigma central de la formación profesional de la Universidad Surcolombiana, la formación por competencias es un discurso pero no una realidad. Por tanto, es necesario que a Universidad defina un modeło pedagógico de formación con el enfoque de competencias que oriente el diseño y la gestión de los procesos curriculares y de evaluación del rendimiento académico. La Universidad es clara al definir el tipo de profesionales que quiere formar por tanto es necesario gue exista una coherencia con ello en los procesos formativos. La Universidad Surcolombiana se está quedando en enunciados solamente creando un distanciamiento entre el discurso y la práctica.

Por lo anterior, es preciso señalar que el proceso evaluativo que se desarrolla en cada uno de los programas académicos de pregrado de la Facultad de Educación no responde a la formación por competencias porque b Universidad no está formando a sus estudiantes bajo este enfoque. No existe ninguna relación entre la evaluación del rendimiento açadémico y el modelo de formación por competencias que senalan los programas de pregrado de la Facultad de Educación en sus Proyectos Educativos, porque es evidente que no estẩn formando a los futuros profesionates de la eduçación desde el enfogue de las competencias.

La evaluación que se realiza en los programas académicos de pregrado de la Facultad de Educación tiene como único fin comprobar los conocimientos y las habilidades gue adquirieron los estudiantes. El juicio que se determina sobre este tipo de evaluación es muy genérico y se da en la medida en que se asigna una calificación a los aprendizajes obtenidos, desconociendo el proceso de aprendizaje de los estudiantes; guiere esto decir que no hay una participación de los mismos en los procesos evaluativos.

Es importante destacar que los directivos de la Universidad Surcolombiana son conscientes de ia problemática y reconocen la importancia de intervenir en la misma. Lo que sucede es que no hay una apropiación del concepto de competencia en la cultura institucional vigente. Los docentes mane jan una concepción restringida que sólo abarca las competencias del saber y del hacer (en el campo de la pedagogía se requieren sujetos con identidad en su oficio profesional, motivados por el trabajo bien hecho, con responsabiildad y disposición de aprender y con idoneidad para saber desempeñarse con excelencia - competencia dei querer), mientras que los estudiantes desconocen por completo el alcance y el vaior de la formación y la evaluación por competencias. Se evidenció que la ausencia de una formación por competencias en la Universidad alude al hecho de gue se presentan vacíos conceptuales y metodológicos que no han permitido que la institución avance en la implementación curricular y didáctica. Al no existir un consenso del concepto de competencia ciaramente definido, no se desarrollan prácticas pedagógicas y evaluativas coherentes con el tipo de profesional que la Universidad pretende formar.

También se presenta esta problemática ante b débil y casi imperceptible decisión y actitud de transformación y cambio del modelo pedagógico tradicional gue no responde a las exigencias básicas del enfoque por competencias, entre cuyas características estructurantes está el saber, el saber hacer en todos los contextos y el querer ser. 
La postura conceptual que tienen los directivos, los docentes y los estudiantes de la Facultad de Educación de la Universidad Surcolombiana frente a la evaluación es muy aproximada a la que se tiene como referente para el desarrollo de la presente investigación, desde la concepción de la evaluación como un proceso que permite realizar seguimiento a las etapas inherentes de los procesos educativos y una fuente de información para conocer los desarrollos, akcances, logros y debilidades de los resultados de la enseñanza. No obstante, es preciso señalar que gran parte de las estrategias e instrumentos de evaluación que se realizan a los estudiantes no responden a esta concepción, lo que quiere decir que la práctica evaluativa de los docentes no es coherente con su discurso conceptual.

Es relevante destacar que la evaluación no es un fin sino un medio para el mejoramiento educativo, por lo cual no puede reducirse a una herramienta de medic ión y control, desde la cual se constata el logro de avances y desarrollos formativos y los resultado de los procesos curriculares y de la naturaleza de las prácticas pedagógicas de formación, de tal forma que permita una revisión y modificación de los mismos.

En la Facultad de Educación no hay una concertación del docente con el estudiante al iniciar l curso en donde se defina qué se va a evaluar, para qué se va a evaluar y cómo se va a evaluar. Es necesario que los estudiantes conozcan cómo van a ser valorados, las estrategias de evaluación, cómo serán, cuándo, con qué recursos, etc;; así mismo, el docente debe propiciar la generación de espacios pedagógicos que busquen resolver situaciones que invoLucren al estudiante dando lugar a la indagación autónoma del mismo desde la base de la duda, la indagación y la solución, situación que nose da sólo a través de la realización de parciales. Es relevante destacar que la evaluación se convierte en el resultado de una comunicación permanente entre docentes y estudiantes, en donde la participación de éste en la construcción de la misma, es fundamental.

Las estrategias de evaluación que actualmente desarrollan los docentes de la Facultad de educación son: La evaluación escrita Las exposiciones Los debates El seminario Los trabajos escritos El trabajo en grupo Las prácticas (de laboratorio, extramuros, pedagógicas) La estrategia que prevalece en cada uno de los programas académicos de la Facult ad es la evaluación escrita con preguntas de selección múltiple que se lleva a cabo a través del parcial como instrumento de evaluación. Quiere esto decir que este tipo de examen evalúa competencias interpretativas (comprensión de información en cualquier sistema de símbolos o formas de representación) y argumentativas (explicación y justificación de enunciados y acciones).

Este tipo de pruebas presenta una contradicción entre el discurso y la realidad del instrumento, pues nose pueden evaluar competencias con preguntas de selección múltiple y no se puede hablar de una evaluación por competencias cuando se realizan unas pruebas con el fin de medir los resultados a partir de contenidos solamente; este tipo de instrumentos no evalúa los niveles más complejos. Quiere esto decir, que se requiere ir más allá, a la intertextualidad, al pensamiento crítico que permita que el estudiante puede confrontar, elaborar pensamiento, fijar su posición; la evaluación debe permitir valorar el desempeño, los intereses, las capacidades y la manera de actuar del estudiante en el contexto académico, laboral y cotidiano, y este propósito en la actualidad no se está logrando. 
Nose puede hablar de una evaluación por competencias cuando se realizan unas pruebas con el fin de medir los resultados, desconociendo la dimensión del ser en el proceso de aprendizaje, el desempeño, los intereses, las capacidades y la manera de actuar del estudiante en el contexto académico, laboral y cotidiano. La evaluación por competencias es formativa y permite valorar el desempeño del eștudiante durante todo el proceso de aprendizaje.

Finalmente, a partir de las anteriores conclusiones, es relevante señalar que es necesario que la Universidad Surcolombiana estructure y adopte el modelo pedagógico sustentado en la indagación sistemática, básico para lograr una formación y evaluación por competencias. No es conveniente ni acertado pensar que un modelo pedagógico de transmisión logre desarrollar las competencias genéricas básicas y las competencias específicas de los profesionales en las diferentes áreas del conocimiento. La presente investigación permitió evidenciar la distancia que se presenta cuando se afirma que se está formando por competencias y lo queen realidad se desarrolla en la práctica; por ende, si se afirma que se forma por competencias se reguiere que modifique o transforme el modelo pedagógico actual de la Universidad Surcolombiana. Por lo anterior se planteó una propuesta alternativa de intervención que permitirá dar solución a la problemática encontrada.

Durante los últimos años, el Grupo de Investigación PACA ${ }^{56}$ a través de su director, el investigador Nelson Ernesto López Jiménez, ha presentado a las instancias directivas académicas y administrativas de la Universidad Surcolombiana una propuesta académica curricular que garantiza una formación por competencias, ya que permite cambiar, transformar y de-construir el modelo pedagógico de transmisión vigente en la Universidad por un modelo soportado en procesos asociados a la integralidad, la transversalidad, la interdisciplinariedad y la transdisciplinariedad. Por lo anterior, a continuación se señalan algunos elementos que permitirăn a la Univer sidad intervenir en la problemática enunciada en las conclusiones:

Se propone que la Universidad Surcolombiana avance en un proceso de de-construcción de los elementos constituyentes de la actual cultura académica. Quiere esto decir que se deben asumir como problemáticas las estructuras adiministrativas, académicas, curriculares, pedagógicas y culturales de tal forma que puedan ser intervenidas, en la perspectiva de alcanzar pertenencia social y pertinencia académica, como base de la calidad y excelencia académicas.

Se plantea que la Universidad Surcolombiana debe cambiar el modelo pedagógico de transmisión por uno que permita que la formación se sustente en un proceso de indagación permanente, en donde su desarrollo genera una concepción diferente de los roles que desempeñan cada uno de los agentes del acto formativo. La formación en esta concepción se entiende como "la sintesis creativa entre el campo de conocimientos y el campo de problemas que generan características determinantes de los procesos a partir de la duda, la incertidumbre y la creación colectiva que su desarrollo comporta" (López Jiménez; 2011), para lo cual se hace relevante que la Universidad se apropie del modelo pedagógico de indagación sistematica-

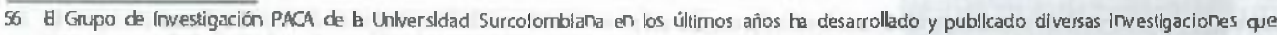

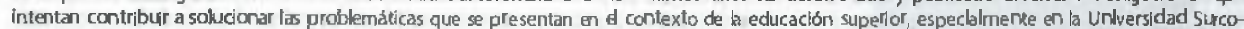

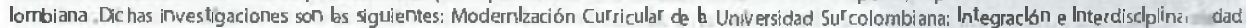
(201 1): Untuersidad y Estilos de Aprendizap (2012); b Universldad y su compromiso de Servkio. Sorial (2012). 
MPIS, propuesto por López Jiménez, ya que éste permite superar la simple asimilación dêl conocimiento y se convierte en una estrategia que garantiza la for mación por competencias a través de la investigación, la capacidad de asombro y la de solución de problemas.

En el modelo pedagógico de indagación sistemática-MPIS los roles de los agentes están determinados por la búsqueda de respuestas satisfactorias a preguntas pertinentes; sus características estân directamente relacionadas con un cambio en la cultura formativa de la Universidad Surcolombiana que afecten los discursos, los procesos ya los agentes involucrados en ef proceso formativo. Es importante señalar que la concepción de docencia, de investigación y de evaluación en este modelo pedagógico recoge la impronta de su estructura, es decir, genera una identidad particular y singular.

Si la competencia es una estructura comple ja que integra el saber, el saber hacer, y el querer o desear hacer, el Modelo Pedagógico de Indagación Sistemática MPIS soportado en la interacción entre un campo de conocimientos y un campo de problemas, garantiza que las condiciones estructurales de la competencia sean logradas a partir del desarrollo det mismo. Este modelo pedagógico desde el enfoque de competencias se convierte en una estrategia asociada a la investigación, al cultivo de la capacidad de asombro, en donde su dinámica está directamente relacionada con la construcción de sentido y con la solución de problemas de toda índole.

El modelo de formación desde el enfoque de competencias supera la relación asimétrica docente-estudiante; los problemas y su solución se convierten en lo esencial de este modelo; el trabajo en equipo es su estrategia metodológica fundamental; la evaluación se convierte en un proceso académico, resultado de la concertación y de los productos alcanzados eliminando la concepción de evaluar desde el "déficit" y resalta los procesos desarrollados y logra en el estudiante propiedades relacionadas con la autonomía, el análisis simbólico, la lectura crítica y propositiva y la innovación permanente (López Jiménez: 2011).

Es relevante señalar que este Modelo Pedagógicode Indagación Sistemática-MPIS garantiza el desarrollo de una evaluación del rendimiento académico por competencias, ya que se relaciona directamente con la necesidad de asumir la evaluación como un "acto de valoraciôn" del trabajo del estudiante con miras a obtener y alcanzar las metas de aprendiza.je. Su finalidad fundamental es la de regular el proceso de enseñanza - aprendizaje de tal forma que permita que las estrategias de formación respondan a las características de los estudiantes ya sus estilos de aprendizaje.

De igual forma, la Universidad Surcolombiana debe realizar cambios en los principios organizativos del conocimiento, ya gue las estructuras curriculares a partir de materias o asignaturas se asocian con la insularidad, la segmentación y la jerarqui zación del conocimiento y su énf asis disciplinario no responde a las emergencias de la actual composición y dinámica del conocimiento. Un proceso de re-estructuración serio y pertinente tiene que intervenir estas formas organizativas, es decir, tiene que intervenir las estructuras de poder y control que la soportan. Los Núcleos Temáticos y Problemáticos que plantea el investigador López Jiménez en el Modelo Pedagógico de Indagación Sistemática-MPIS se constituyen en una alternativa curricular viable para superar la realidad anteriormente aludida. 
El núcleo temático y problemático- NTP, es una estrategía alternativa que pretende integrar la teoría con la práctica, la docencia, la investigación y la proyección social; los saberes académicos, científicos, tradicionales, cotidianos, culturales, tecnológicos; el trabajo individual con el trabajo colectivo y, fundamentalmente, el trabajo autónomo del equipo de estudiantes y docentes.

También se deben realizar cambios en las prácticas pedagógicas de evaluación, ya que éstas, desde el Modelo Pedagógico de Indagación Sistemática-MPIS, se fundamentan estructuralmente en la Pedagogía Crítica que permite convertir las potencialidades y las capacidades de los estudiantes en competencias, y desarrolla estrategias cognitivas, potestativas, actitudinales y de ejecución en contextos variados y deseados o anhelantes para que el estudiante de pregrado encuentre y construya sus propias maneras de aprendiza je y organice sus procesos mentales de acuerdo con sus intereses y metas específicas.

De igual forma, el Modelo Pedagógico de Indagación Sistemática-MPIS permite generar cambios en las prácticas evaluativas del docente yen su estruc tura mental, ya que este modelo exige que a práctica educativa desborde el piano instrumental, de simple desarrollo y ejecución de procedimientos y se caracterice como un proceso social y cultural que comportan las particularidades del contexto, es necesario que la Universidad Surcolombiana garantice a sus docentes una formación y capacitación continuas del nuevo modelo de formación que permita que los docentes se apropien de elementos y factores que faciliten su desempeño con idoneidad y superen los modelos mentales que actualmente agencian, y de esta forma garantizar una relación integral y coherente entre el currículo, las prácticas pedagógicas y la evaluación.

Finalmente la Universidad Surcolombiana debe avanzar en un proceso de detección de necesidades, con el propósito de indagar de una manera compartida los problemas, ya sean de carácter social, académico, investigativo, axiológico, etc., y de esta manera contar con información sólida y pertinente sobre las necesidades y problemas con los cuales van a interactuar los programas académicos, y desde esta base se pueda definir el propósito y el perfil de formación, que se convierte en la misión o norte de cada programa. Es relevante destacar que el propósito de formación es el resultado de acciones de participación, cooperación, negociación y concertación, y por tanto, es objeto de un permanente análisis y cuestionamiento en la medida en que se convierte en referente esencial del proceso formativo. Por lo anterior, su construcción requiere b participación de los diferentes agentes que intervienen en la acción formativa creando un clima democrático en donde los participantes puedan expresarse y definir aspectos relacionados con la visión y el compromiso de cada uno de ellos.

La propuesta de intervencion planteada por el investigador Nelson Ernesto López liménez garantiza que se dé una formación por competencias y por ende una evaluación del rendimiento académico por competencias, permitiendo que exista una relación integral y coherente entre el currículo, las prácticas pedagógicas y la evaluación. La presente investigación se enmarca en el Programa de Investigación del PACA, en la medida que su autora es investigadora activa del mismo y que dada su preocupación investigativa se convierte en un elemento más de los avances investigativos del Grupo de Investigación. 


\section{REFERENCIAS BIBLIOGRÁFICAS:}

Bogoya Maldonado, D. (editor). (2003). Trazas y Miradas: evaluación y competencias. Capitulo II. Universidad Nacional de Colombia.

Bogoya Maldonado, D. (2000). Una prueba de evaluación de competencias académicas como proyecto. En D. Bogoya et al. (Eds.), Competencias y proyecto pedagógico (7-29). Bogotá: Universidad Nacional de Colombia.

Brown, 5. y Glasner, A. (2007). Evaluaren la Universidad. España: Narcea, 5.A. De Ediciones.

Cabra, F. (2008). La evafuación y el enfoque de competencias: Tensiones, limitaciones y oportunidades para la innovación docente en la univer sidad. Colombia: Revista Escuela de Administración de Negocios No. ó3, 91-10ó.

Camperos, M. (2008). La Evaluación por Competencias, mitos, peligros y desafíos. (en línea). Disponible en: <http://www.scielo.org.ve/scielo.php?script=sci_arttext\&pi$d=S 1316-49102008000400017 \& l n g=e s \& n r m=i s 0>$. ISSN 1310 - 4910 .

(Rescatado el 12 de enero de 2012)

Cano, M. (2008). La evaluación por competencias en la educación superior. (en línea). Disponible en: http://www.ugr.es/local/recfpro/rev123COL1.pdf

(Rescatado el 10 de octubre de 2012).

Cerda, H. (2000). La evaluación como experiencia total. Santafé de Bogotá: Magisterio.

Clavijo, Galo. (2008). La evaluación del proceso de formación. Cartagena de Indias. Delors, Jaques (2006). La educación encierra un tesoro: Informe a la UNESCO de la Comisión Internacional sobre la educación para ef siglo XXI, presidida por JACQUES DELORS. UNESCO: Santillana Ediciones, Pág. 95-96.

Díaz Barriga, Á. (2005). El profesor de educación superior frente a las demandas de los nuevos debates educativos. Perfiles Educativos, 27(108), 20.

DfAZ VILLA, Mario. (2001). Del discurso pedagógico: Problemas críticos. Poder, Contr ol y Discurso Pedagógico. Bogotá D.C: Colección SEMINARI UM- MAGISTERIO.

Gette, 5. (2007). Fundamentación Teórica de la Evaluación de los Aprendizajes a Nivel Superior. Barranquilla, Colombia: Editorial Uniautónoma.

Jurado, F. (2003). Trazas y miradas - evaluación y competencias. Bogotá: Unilibros.

Kornblit, Ana L (2007). Metodologias Cualitativas en Ciencias Sociales: Modelos yprocedimientos de análisis. Segunda edición. Buenos Aires: Biblos.

López Jiménez, N. (2011) Modernización curricular de la Universidad Surcolombiana: integración e interdisciplinariedad. Neiva: Editorial Universidad Surcolombiana, Primera edición. 
López, F.B.S. y Hinojos, K. (2002). Evaluación del aprendiza je. Alternativas y nuevos desarrollos. México: Trillas.

López Jiménez, N. (1995). La reestructuración curricular de la Educación Superior: Hacia la integración del saber. Bogotá: ICFES.

López Jiménez, N. (2006). Evaluación por competencias: Un reto por enfrentar y un proceso por construir. Neiva: Editorial Universidad Surcolombiana.

López Jiménez, N. (2013). Universidad y estilos de aprendizajes. Neiva: Editorial Universidad Surcolombiana.

Maldonado, Miguel Ángel (2006). Competencias, métodos y genealogía. Pedagogíaydidáctica del trabajo. Bogotá: ECO EDICIONES, Primera edición.

Mayorga, Román (1999). Los desafios a ka universidad latinoamericana en el siglo XXI. Colombia: Revista lbero Americana de Educación, Número 21.

Ministerio de Educación Nacional. (2010). Evaluación en el aula. (en línea). Disponible en: http://www.mineducacion.gov.co/1621/article-236979.html.

(Recuperado el 23 de agosto de 2012).

Ministerio de Educación Nacional. Decreto 1295 de 2010. Porel cual se reglamenta el registro calificado de que trata la Ley 1188 de 2008 y la of erta y desarrollo de programas académicos de educación superior. Bogoț́a: MEN.

Rodriguez, T, Álvarez, L., Hernández, J., Muñiz, J. Y Soler, E (2000). Evaluación en el aula. España: Ediciones Nobel.

Sally, B. y Glasner, A. (2007). Evaluar en la Universidad-problemas y nuevos enfogues. España: Narcea, S.A. de Ediciones.

Serrano Orejuela, E. (2003). B concepto de competencia en la semántica Discursiva. Colección Cuadernos del Seminario en Educación, No. 2. Instituto de Investigación en Educación. Bogotá: Universidad Nacional.

Tobón, Sergio. (2006). Competencias en la educación superior-Politicas hacia la calidad. Bogotá: ECOE EDICIONES.

Tobón, Sergio. (2008). La formación basada en competencias en la educación superior: el enfoque comprejo. Universidad Autônoma de Guadalajara. México: CURSO झGLU.

Universidad Surcolombiana. (2009). Acuerdo Número 0020 de 2003. Por el cual se adopta el Proyecto Educativo Universitario. Neiva - Huila.

Vasco, Carlos E. (2005). ¿Logros, objetivos o indicadores de evaluación? Colombia: Ministerio de Educación Nacional. 


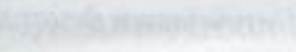

1.

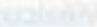

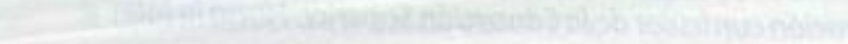

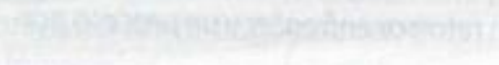

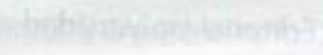

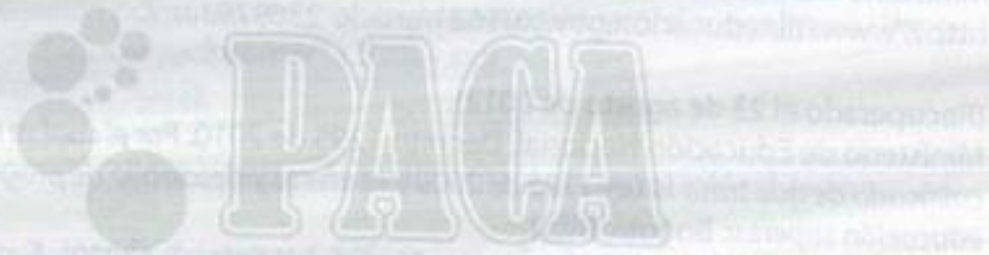

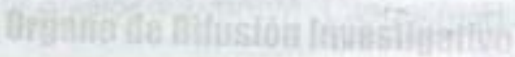

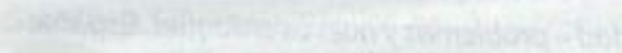

